\title{
Association between the intima-media thickness of the brachiocephalic trunk and white matter hyperintensity in brain MRI
}

\begin{abstract}
Katsunori Isa $^{1}$, Atsushi Sakima ${ }^{1}$, Hirokuni Sakima ${ }^{1}$, Koh Nakachi ${ }^{1}$, Kozen Kinjyo ${ }^{1,2}$ and Yusuke Ohya ${ }^{1}$
The intima-media thickness (IMT) of the brachiocephalic trunk (BCT) can be measured using duplex carotid ultrasonography, which is used for imaging the common carotid artery (CCA). However, the clinical significance of the BCT-IMT has not been studied. We reviewed 1109 stroke-free participants in the registry of the Okinawa General Health Maintenance Association. We compared the association between the BCT-IMT or the CCA-IMT with deep and subcortical white matter hyperintensity (DSWMH). The BCT-IMT was correlated with the CCA-IMT, and like CCA-IMT, it increased with advancing age. The increase in both the BCT-IMT and the CCA-IMT quartiles was correlated with the development of DSWMH. The multivariate logistic regression analysis indicated that, as observed for the CCA-IMT, the increase in the BCT-IMT was associated with a higher prevalence of significant DSWMH (Fazekas grade 2 or 3 per $0.1 \mathrm{~mm}$ increase in IMT; OR $1.02,95 \%$ confidence interval $1-1.04 ; P=0.04)$. The increase in quartiles of the BCT-IMT was only associated with a higher prevalence of significant DSWMH in subjects with lower CCA-IMT ( 1 st and 2 nd quartiles, $R^{2}=0.18, P<0.05$ ) but not in subjects with higher CCA-IMT (3rd and 4th quartiles). Combinations of the CCA-IMT and BCT-IMT quartiles failed to have an additive effect on the prevalence of significant DSWMH. The BCT-IMT has a similar clinical profile to the CCA-IMT in terms of its association with DSWMH. However, the CCA-IMT and the BCT-IMT did not predict DSWMH in an additive manner, and distinct mechanisms might underlie the observed thickening of the IMT in the CCA and BCT. Hypertension Research (2013) 36, 980-984; doi:10.1038/hr.2013.72; published online 11 July 2013
\end{abstract}

Keywords: brachiocephalic trunk; common carotid artery; intima-media thickness; white matter hyperintensity

\section{INTRODUCTION}

The brachiocephalic trunk (BCT) is the first branch of the aortic arch. Shortly after emerging, it divides into the right common carotid artery and the right subclavian artery. A ruptured plaque in the BCT can form a thrombus, which has been reported to cause ischemic stroke. ${ }^{1,2}$ The intima-media thickness (IMT) of the BCT (BCT-IMT) can be measured by using duplex carotid ultrasonography, which is used for imaging the common carotid artery (CCA). ${ }^{3}$ However, the clinical significance of the BCT-IMT in predicting silent ischemic lesions of deep and subcortical white matter (DSWMH) has not been studied. ${ }^{4,5}$ The purpose of this study is to address the clinical profile of the BCT-IMT and its association with DSWMH.

\section{METHODS}

Subjects

This was a retrospective, cross-sectional study using data obtained from healthscreening tests, specifically those relating to the brain, in Japan. We reviewed 1109 participants listed in the registry of the Okinawa General Health
Maintenance Association (OGHMA) between May 2006 and March 2011, who had no previous disability by stroke and whose BCT-IMT data were available. This retrospective study was approved by the Institutional Ethics Committee of Okinawa General Health Maintenance Association and University of the Ryukyus.

Cerebrovascular and cardiovascular risk indicators

Trained nurses of the Okinawa General Health Maintenance Association obtained past history, medication, any smoking histories, body mass index (BMI), systolic and diastolic blood pressure (SBP, DBP) and a mini-mental state examination (MMSE) from the participants. The blood pressure of the participants was measured using a conventional sphygmomanometer. Total cholesterol (Tchol), triglyceride (TG), high-density lipoprotein cholesterol (HDL-C), low-density lipoprotein cholesterol (LDL-C), blood urea nitrogen (BUN), creatinine (Cre), uric acid (UA), and blood glucose and hemoglobin Alc (HbAlc, JDS) were also measured. The estimated glomerular filtration rate (eGFR, $\mathrm{ml} \mathrm{min}^{-1} 1.73 \mathrm{~m}^{-2}$ ) was calculated using a formula standardized for the Japanese population, $0.741 \times 175 \times \mathrm{Age}^{-0.203} \times \mathrm{Cre}^{-1.154}(\times 0.742$, if female).

${ }^{1}$ Department of Cardiovascular Medicine, Nephrology and Neurology, University of the Ryukyus Graduate School of Medicine, Nishihara, Japan and ${ }^{2} \mathrm{Okinawa}$ General Health Maintenance Association, Haebaru, Japan

Correspondence: Dr K Isa, Department of Cardiovascular Medicine, Nephrology and Neurology, University of the Ryukyus Graduate School of Medicine, 207 Uehara, Nishihara, Okinawa 903-0215, Japan.

E-mail: kisaneurology@gmail.com

Received 7 January 2013; revised 7 March 2013; accepted 3 April 2013; published online 11 July 2013 


\section{Measurement of IMT}

To measure the carotid IMT, we performed duplex ultrasonography of the CCA or the BCT using Prosound $\alpha 7$ (Aloka, Tokyo, Japan) with a $6.7 \mathrm{MHz}$ linear-array transducer. In a longitudinal image of the artery on ultrasonographic B-mode, the posterior (far) walls of the carotid artery were displayed as two bright, white lines separated by a hypoechogenic space. The maximum distance between the leading edge of the first bright line of the far wall and the leading edge of the second bright line corresponded to the IMT. The IMT in the CCA far wall (CCA-IMT) was measured in a $10-\mathrm{mm}$ proximal section from the artery to the end of the carotid bulb on both the sides. The IMT in the BCT (BCT-IMT) was defined as the BCT far wall within visible range or $\leqslant 2 \mathrm{~cm}$ from the aortic side of the right common carotid artery branch (Figure 1). Recording and measuring of both CCA-IMT and BCT-IMT were performed by a single sonographer who was blinded to any other medical information about the participants.

\section{The brain magnetic resonance imaging}

All participants were examined on the 1.5-Tesla MRI unit (Siemens Magnetom Symphony Syngo; Siemens AG, Munich, Germany). The deep and subcortical white matter hyperintensity (DSWMH) was diagnosed on the FLAIR axial image of the brain MRI. The severity of DSWMH was rated visually using the Fazekas scale, which assigns grades ranging from 0 , which corresponds to normal, to $1-3$, depending on the severity. A single person performed the visual ratings of DSWMH in a blinded manner.

\section{Statistical analysis}

The data values were presented as $n(\%)$ or median (inter-quartile range, IQR). Pearson's or Spearman's correlation coefficients were used for continuous or ordinal variables, respectively. Parameter comparisons between subgroups were made using Student's $t$-test. The multivariate linear regression analysis was carried out to check the independent relationships between CCA-IMT or BCT-IMT and the Fazekas grade of DSWMH. A P-value of less than 0.05 was considered to be statistically significant. All statistical analyses were performed using JMP 9 software (SAS Institute Inc., Cary, NC, USA).

\section{RESULTS}

\section{Baseline characteristics}

The baseline characteristics of participants are shown in Table 1. Fiftyfour percent of the participants were male individuals, 15\% were 65 years or older, $4 \%$ had history of heart disease, $2 \%$ had history of the kidney disease and $37 \%$ had any smoking histories. A total of $22 \%$ of participants reported current treatment with an antihypertensive drug, $4 \%$ with antidiabetic drugs and $11 \%$ with hyperlipidemic drugs. Most participants obtained full scores on the cognitive test, and only $2 \%$ of them scored $<27$ in the MMSE. DSWMH was found in $34 \%$ of participants (grade 1, 10\%; 2, 21\%;3,3\%).

\section{Intima-media thickness}

The BCT-IMT (median 1.1, IQR 0.9-1.4 mm) was similar to the CCA-IMT (1.0, 0.8-1.4) and also showed an increase with advancing age, similar to the CCA-IMT (Figure 2). As such, the BCT-IMT was correlated with the CCA-IMT $\left(R^{2}=0.089, P<0.0001\right)$.

Association with cerebrovascular and cardiovascular risk indicators The associations between cardiovascular risk factors and IMT are shown in Table 2. The CCA-IMT was significantly associated with all risk indicators. Specifically, it was negatively associated with eGFR and HDL-C and positively associated with other risk indicators. The BCT-IMT was significantly associated with age, SBP, Tchol, LDL-C, eGFR, BUN, blood glucose and HbA1c but not with BMI, DBP, TG, HDL-C, Cre and UA. The CCA or BCT-IMT and the Fazekas grade $\left(R^{2}=0.07, P<0.0001\right)$ were predominantly correlated with age, rather than with other cardiovascular risk factors.

\section{Associations between IMT and DSWMH}

The increase in either the BCT-IMT or the CCA-IMT quartiles was correlated with the development of DSWMH (the BCT-IMT, $R^{2}=0.01, P=0.001$; the CCA-IMT, $R^{2}=0.02, P<0.001$, Figure 3$)$.

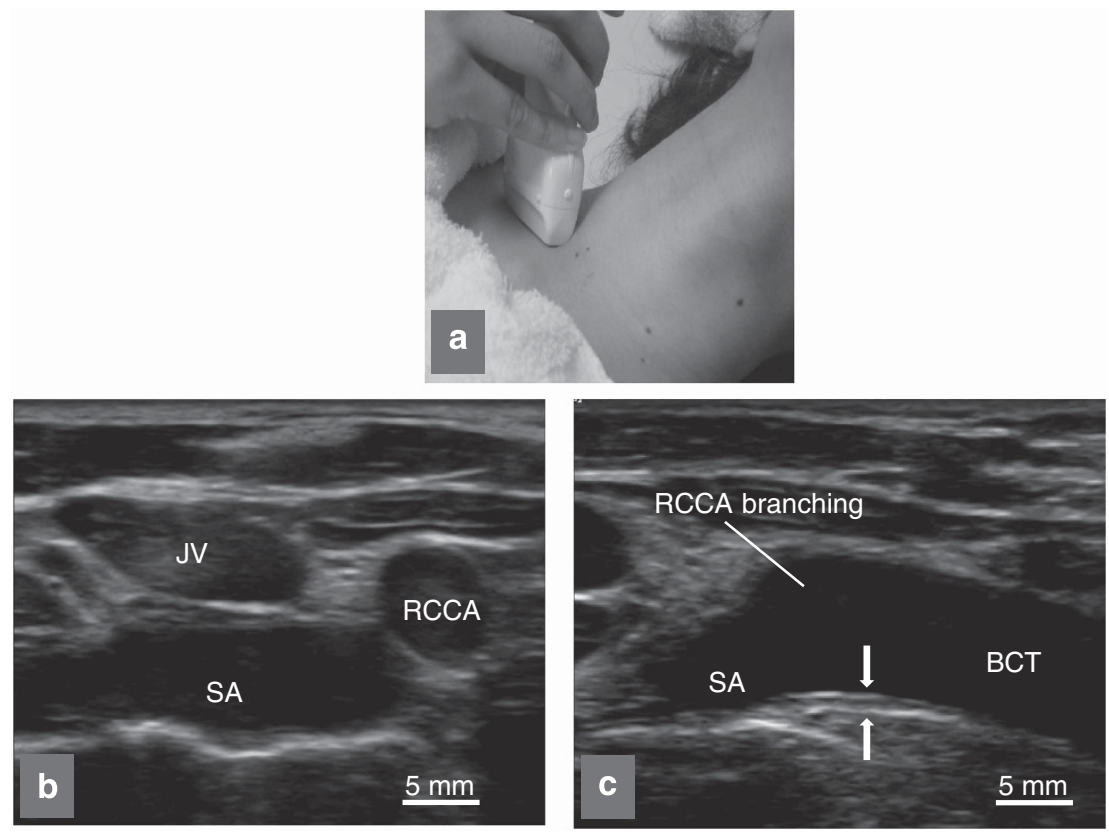

Figure 1 The ultrasonographic examination of the brachiocephalic trunk (BCT). (a) Transducer position; (b) subsequent B-mode picture of the right common carotid artery (RCCA), jugular vein (JV) and subclavian artery (SA); (c) positioning the transducer in a more caudal location enables the visualization of the BCT. The BCT-IMT was defined as max-IMT of the BCT far wall (arrows) within visible range or $\leqslant 2 \mathrm{~cm}$ from the aortic (medial) side from RCCA branching. A full color version of this figure is available at the Hypertension Research journal online. 
Table 1 Baseline characteristics, $n=1109$

\begin{tabular}{|c|c|c|}
\hline Male & $617(56)$ & \\
\hline Age, years & $54(44-60)$ & \\
\hline Range & $24-85$ & \\
\hline Age $\geqslant 65$ years & $166(15)$ & \\
\hline Smoking history & $408(37)$ & \\
\hline \multicolumn{3}{|l|}{ Past history } \\
\hline Heart disease & $42(4)$ & \\
\hline Kidney disease & $19(2)$ & \\
\hline \multicolumn{3}{|l|}{ Medication } \\
\hline Antihypertensive & $245(22)$ & \\
\hline Antidiabetic & $49(4)$ & \\
\hline Antihyperlipidemic & $122(11)$ & \\
\hline $\mathrm{BMI}, \mathrm{kg} \mathrm{m}^{-2}$ & $24(22-26)$ & \\
\hline $\mathrm{SBP}, \mathrm{mm} \mathrm{Hg}$ & $120(110-130)$ & \\
\hline $\mathrm{DBP}, \mathrm{mm} \mathrm{Hg}$ & $76(70-82)$ & \\
\hline MMSE & $30(30-30)$ & \\
\hline MMSE <27 & $23(2)$ & \\
\hline Tchol, mgdl-1 & $204(182-227)$ & \\
\hline TG, mgdl-1 & $100(71-150)$ & \\
\hline HDL-C, mgdl-1 & $57(48-68)$ & \\
\hline LDL-C, $\mathrm{mg} \mathrm{dl}^{-1}$ & $121(100-141)$ & \\
\hline eGFR, $\mathrm{ml} \mathrm{min}^{-1} 1.73 \mathrm{~m}^{-2}$ & $72(65-81)$ & \\
\hline BUN, mgdl-1 & $13(11-16)$ & \\
\hline Cre, $\mathrm{mgdl}^{-1}$ & $0.8(0.7-0.9)$ & \\
\hline $\mathrm{UA}, \mathrm{mgdl}-1$ & $5.8(4.8-6.7)$ & \\
\hline Blood glucose, $\mathrm{mg} \mathrm{dl}^{-1}$ & $96(91-103)$ & \\
\hline HbAlc (JDS), \% & $5.1(4.9-5.4)$ & \\
\hline Intima-media thickness (IMT, mm) & BCT-IMT & CCA-IMT \\
\hline Mean & 1.2 & 1.2 \\
\hline Median & 1.1 & 1.0 \\
\hline Range & $0.4-5.4$ & $0.5-4.2$ \\
\hline IQR & $0.9-1.4$ & $0.8-1.4$ \\
\hline \multicolumn{3}{|c|}{ Fazekas grade, DSWMH on the brain MRI } \\
\hline Grade 0 & 727 (66) & \\
\hline Grade 1 & $107(10)$ & \\
\hline Grade 2 & $241(21)$ & \\
\hline Grade 3 & $34(3)$ & \\
\hline Microbleeds & $28(3)$ & \\
\hline
\end{tabular}

Abbreviations: BCT, brachiocephalic trunk; BMI, body mass index; BUN, blood urea nitrogen; CCA, common carotid artery; Cre, creatinine; DBP, diastolic blood pressure; DSWMH, deep and subcortical white matter hyperintensity; eGFR, estimated glomerular filtration rate; HDL-C, high-density lipoprotein cholesterol; LDL-C, low-density lipoprotein cholesterol; MMSE, minimental state examination; SBP, systolic blood pressure; Tchol, total cholesterol; TG, triglycemia UA, uric acid.

Values are presented as $n(\%)$ or median (IQR).

The multivariate logistic regression analysis indicated that the increase in the BCT-IMT was associated with a higher prevalence of significant DSWMH (Fazekas grade 2 or 3 per $0.1 \mathrm{~mm}$ increase in IMT; OR 1.02, 95\% CI 1-1.04; $P=0.04$, Table 3 ) when adjusted for sex, BMI, SBP, eGFR, Tchol and HbAlc. This was also the case for the CCA-IMT (OR 1.05, 95\% CI 1.03-1.08, $P<0.001$ ). However, if the adjustment included age (model 2), no association was found between increasing CCA-IMT or BCT-IMT and a higher prevalence of significant DSWMH. The increase in quartiles of the BCT-IMT was only associated with a higher prevalence of significant DSWMH in subjects with lower CCA-IMT ( 1 st and 2nd quartiles, $R^{2}=0.18, P<0.05$ ) but not in subjects with higher CCA-IMT (3rd and 4th quartiles, $\left.R^{2}=0.005, P=0.3\right)$. In addition, combinations of the CCA-IMT

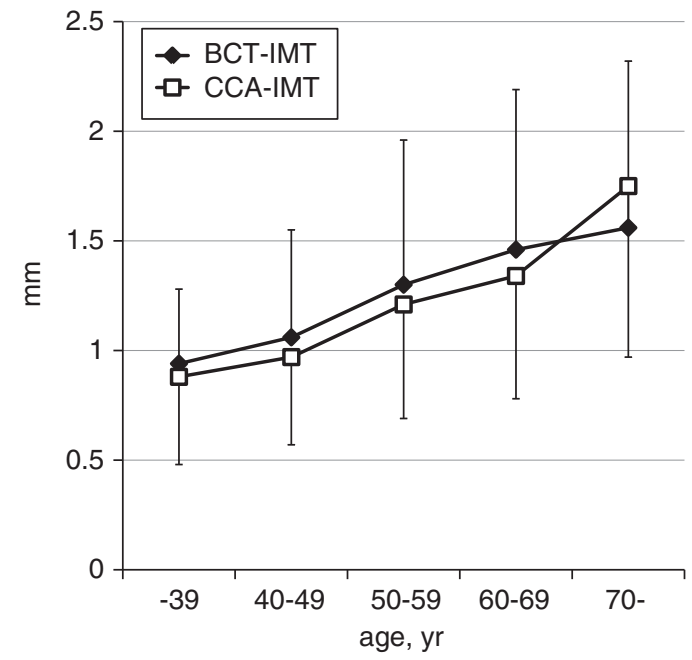

Figure 2 The trends of change in IMT for each age class. BCT, brachiocephalic trunk; CCA, common carotid artery.

and BCT-IMT quartiles failed to have an additive effect in increasing the prevalence of significant DSWMH (Figure 4).

\section{DISCUSSION}

In this study, we addressed the clinical profile of BCT-IMT and its association with DSWMH in comparison with the CCA-IMT. The BCT-IMT was similar to the CCA-IMT in clinical profile and showed a similar increase with advancing age. In addition, as with the CCAIMT, the BCT-IMT was associated with cardiovascular risk factors, and the increase in BCT-IMT quartiles was also associated with the development of DSWMH.

White matter hyperintensity in the brain MRI is frequently observed in elderly participants without apparent neurological symptoms. A recent systematic review showed that white matter hyperintensity in the brain magnetic resonance imaging predicts an increased risk of stroke, dementia and death. ${ }^{6}$ Therefore, white matter hyperintensity can be a screening indicator for risk factors of stroke and dementia. In addition, the carotid IMT is a strong predictor of future vascular events, myocardial infarction or stroke. ${ }^{4}$ The increases in carotid IMT have been shown to be directly associated with an increased risk of myocardial infarction and stroke in older adults without a history of cardiovascular disease. ${ }^{7}$ As was described in the CAPS study, ${ }^{8}$ the predictive value of carotid IMT in younger subjects ( $>45$ years) is at least as high as that in older subjects. The BCT-IMT has previously been shown to be positively associated with DSWMH, as well as the CCA-IMT. ${ }^{5,9}$ When taken together, the BCT-IMT may be worth considering as a possible surrogate maker of stroke and dementia.

This study failed to show the superiority of BCT-IMT over the CCA-IMT and age in its correlation with the progression of DSWMH. The multiple logistic regression analysis shown in Table 3 suggests that age was the strongest confounding factor between IMT and the Fazekas grade. Although the BCT-IMT has a similar clinical profile to the CCA-IMT in terms of its association with DSWMH, combinations of the CCA-IMT and BCT-IMT quartiles were not helpful for building a better association for significant DSWMH.

Notably, even in subjects with low CCA-IMT (1st and 2nd quartiles) and high BCT-IMT, the prevalence of significant DSWMH was high. Subjects with higher CCA-IMT (3rd and 4th quartiles) had a higher prevalence of significant DSWMH regardless of the 
Table 2 Associations between cardiovascular risk factors and IMT

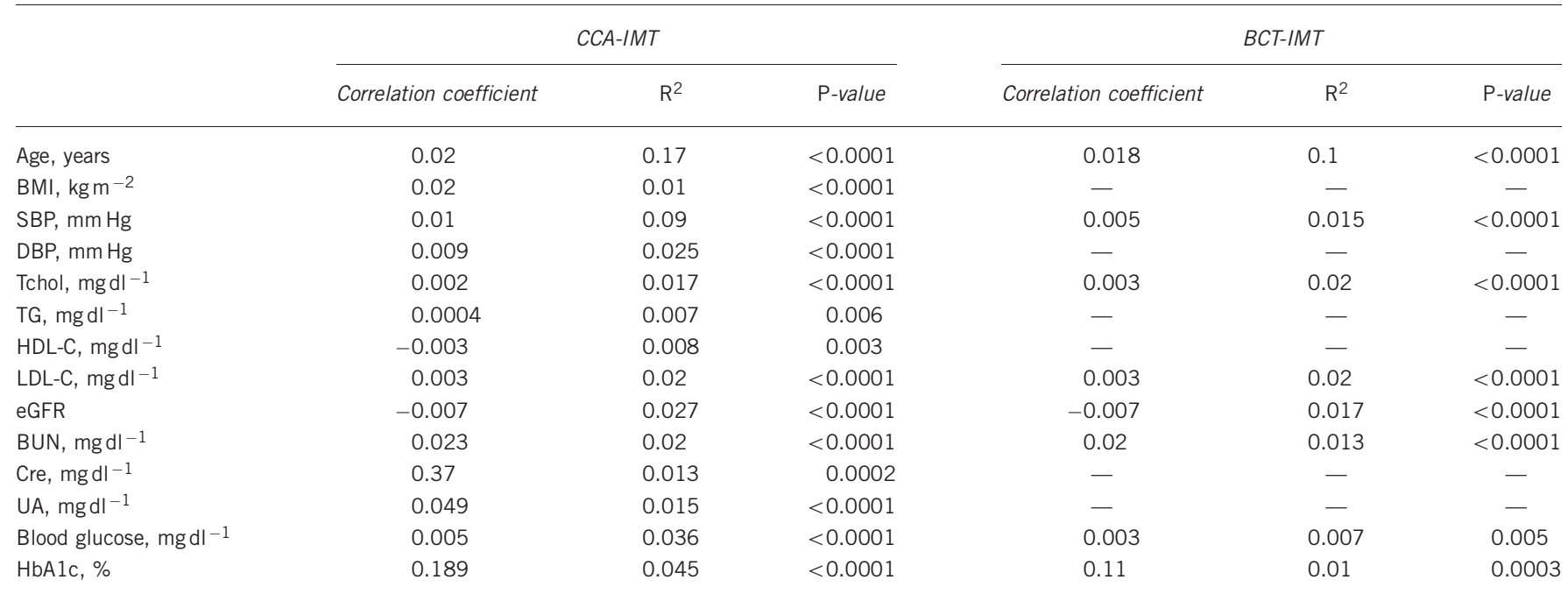

Abbreviations: BCT, brachiocephalic trunk; BMI, body mass index; BUN, blood urea nitrogen; CCA, common carotid artery; Cre, creatinine; DBP, diastolic blood pressure; DSWMH, deep and subcortical white matter hyperintensity; eGFR, estimated glomerular filtration rate; HDL-C, high-density lipoprotein cholesterol; LDL-C, low-density lipoprotein cholesterol; MMSE, mini-mental state examination; SBP, systolic blood pressure; Tchol, total cholesterol; TG, triglycemia; UA, uric acid.
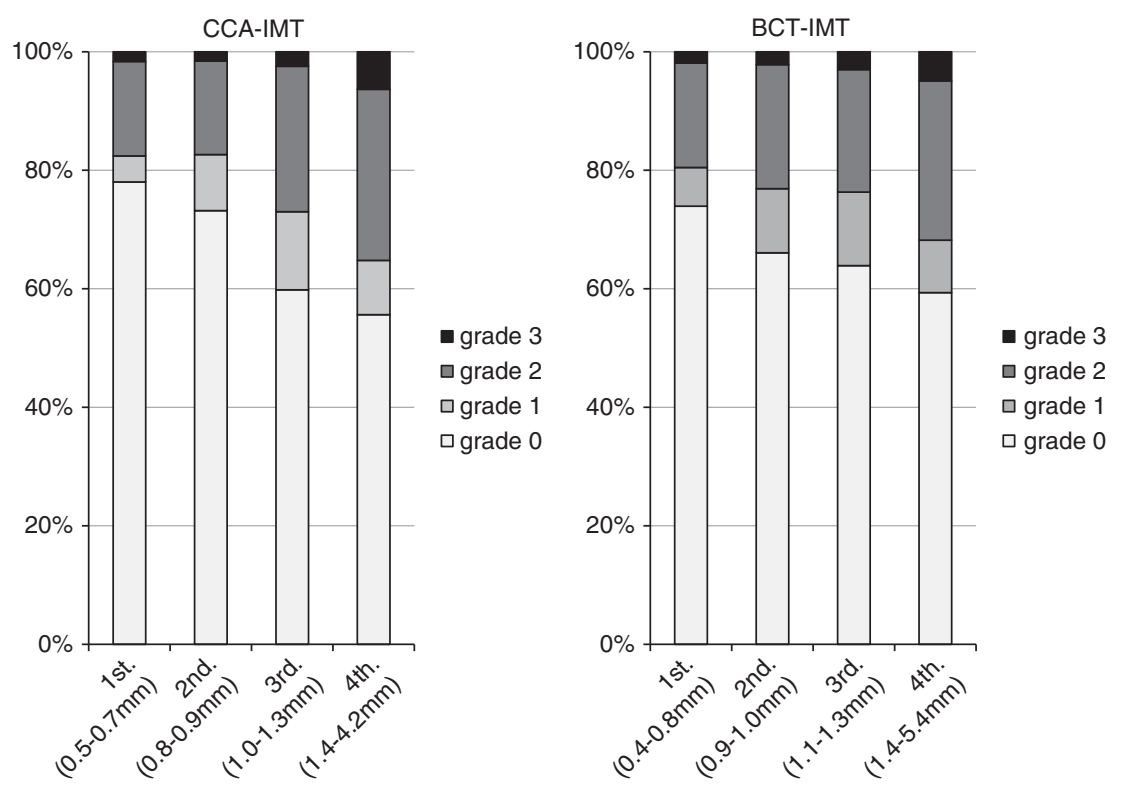

Figure 3 The relationship between deep and subcortical white matter hyperintensity of Fazekas grade and IMT quartiles. A significant association between Fazekas grade and IMT quartiles was found both in the BCT $\left(R^{2}=0.01, P=0.001\right)$ and CCA $\left(R^{2}=0.02, P<0.001\right)$.

Table 3 The multiple logistic regression analysis for DSWMH grade 2 or 3 by increase per $0.1 \mathrm{~mm}$ in IMT

\begin{tabular}{|c|c|c|c|c|c|c|}
\hline & \multicolumn{2}{|c|}{ Unadjusted } & \multicolumn{2}{|c|}{ Model 1} & \multicolumn{2}{|c|}{ Model 2} \\
\hline & OR $(95 \% \mathrm{Cl})$ & P-value & OR $(95 \% \mathrm{Cl})$ & P-value & OR $(95 \% \mathrm{Cl})$ & P-value \\
\hline BCT-IMT & $1.03(1.01-1.05)$ & 0.002 & $1.02(1-1.04)$ & 0.04 & $1.00(0.97-1.02)$ & 0.9 \\
\hline
\end{tabular}

Abbreviations: BCT, brachiocephalic trunk; BMI, body mass index; BUN, blood urea nitrogen; CCA, common carotid artery; Cre, creatinine; DBP, diastolic blood pressure; DSWMH, deep and subcortical white matter hyperintensity; eGFR, estimated glomerular filtration rate; HDL-C, high-density lipoprotein cholesterol; LDL-C, low-density lipoprotein cholesterol; MMSE, mini-mental state examination; SBP, systolic blood pressure; Tchol, total cholesterol; TG, triglycemia; UA, uric acid.

Model 1 is adjusted by sex, BMI, SBP, eGFR, Tchol and HbAlc. Model 2 is adjusted by sex, BMI, SBP, eGFR, Tchol, HbAlc and age. 


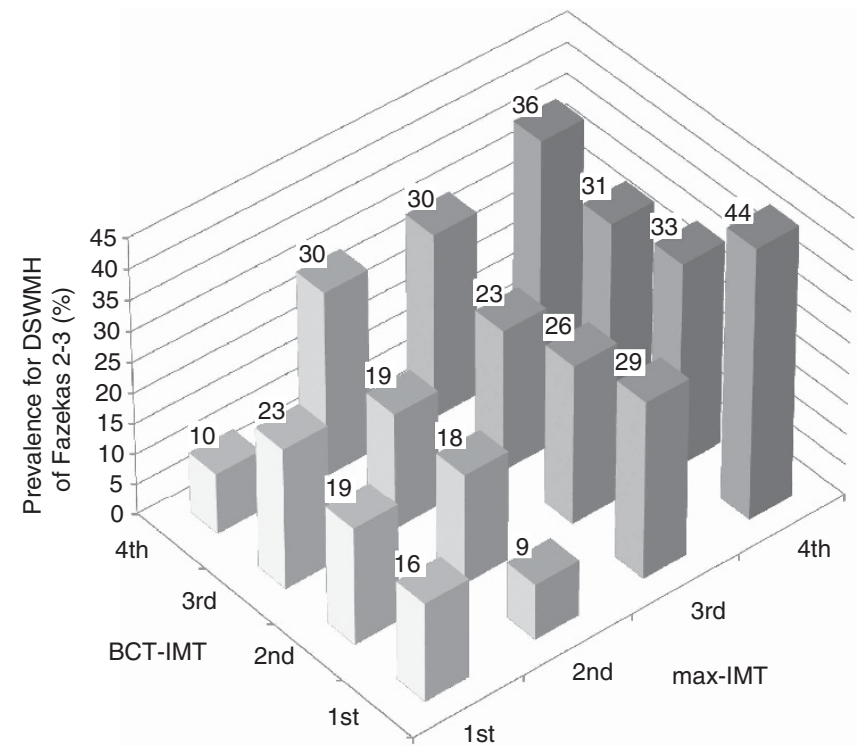

Figure 4 The prevalence of Fazekas grade 2-3 of DSWMH by BCT-IMT and CCA-IMT quartiles. The increase in quartiles of the BCT-IMT was only associated with a higher prevalence of significant DSWMH in subjects with lower CCA-IMT (1st and 2nd quartiles, $R^{2}=0.18, P<0.05$ ), but not in subjects with higher CCA-IMT (3rd and 4th quartiles, $R^{2}=0.005, P=0.3$ ).

BCT-IMT. For practical use, the additional determination of BCTIMT may only be useful for stratifying the risk of significant DSWMH if the subject has low CCA-IMT. Given these observations, distinct mechanisms might be involved in the thickening of IMT of the CCA and BCT, even though this argument needs to be confirmed by future prospective studies with a larger number of subjects. The BCT is the first branch of the aortic arch and can be affected to a greater extent by central blood pressure, which progressively weakens with the distance from the aorta, than the CCA. The distending pressure in the larger, elastic-type arteries is a key determinant of the degenerative changes that characterize accelerated aging and hypertension. In contrast, the muscular peripheral arteries, such as the brachial and the radial arteries, are less influenced by these changes. ${ }^{10}$ Central pressures are pathophysiologically more relevant than peripheral (brachial) pressures in the pathogenesis of cardiovascular disease. ${ }^{11}$ Specifically, in earlier stages, central SBP has been shown to correlate more closely with measures of cardiovascular disease risk and DWMH than brachial SBP. ${ }^{5}$ Assessing the correlation between the BCT-IMC or CCA-IMT and central pressure or brachial pressure will be expected to address the clinical significance of the BCT-IMT as a surrogate marker for cardiovascular diseases.

This study had some limitations. First, a method for measuring the BCT-IMT has not been previously established in other studies. At this time, we used the most simple and reliable method of BCT-IMT measurement available; however, this should be addressed in future studies. Second, the retrospective and cross-sectional study design involving relatively healthy participants limited the statistical power for addressing the differences between BCT-IMT and CCA-IMT in their clinical profiles and their association with DSWMH. Additional longitudinal observation studies are needed to establish the clinical importance of the IMTs of these two arteries.

In this study, we studied generally healthy, middle-aged Japanese to evaluate the baseline characteristics of BCT-IMT. In the future, we will expand our study to include patients with stroke, ischemic heart disease and/or peripheral arterial disease to assess the utility of BCTIMT as a surrogate marker for cardiovascular events and dementia.

In conclusion, the BCT-IMT has a similar clinical profile to the CCA-IMT in terms of its association with DSWMH. However, the CCA-IMT and BCT-IMT did not predict DSWMH in an additive manner, and, as such, distinct mechanisms might be involved in the thickening of the IMT in the CCA and BCT. Further prospective studies with wider perspectives will be needed to identify the clinical utility of measuring BCT-IMT as a surrogate marker for cardiovascular diseases.

\section{CONFLICT OF INTEREST}

The authors declare no conflict of interest.

1 Youn SW, Yu SW, Lee NJ, Kim JH. Neurological picture. Acute middle cerebral artery stroke and an innominate steal from a ruptured brachiocephalic trunk atheroma. I Neurol Neurosurg Psychiatry 2009; 80: 492-493.

2 Nakajima M, Yasaka M, Minematsu K. Mobile thrombus from a ruptured plaque in the brachiocephalic artery. J Stroke Cerebrovasc Dis 2008; 17: 423-425.

3 Bartes E. Subclavian Artery and Brachiocephalic Trunk; Color-coded Duplex Ultrasonography of Cerebral Vessels Atlas and Manual. Schattauer, Stuttgart, 1999, pp 157-172.

4 Lorenz MW, Markus HS, Bots ML, Rosvall M, Sitzer M. Prediction of clinical cardiovascular events with carotid intima-media thickness: A systematic review and meta-analysis. Circulation 2007; 115: 459-467.

5 Shrestha I, Takahashi T, Nomura E, Ohtsuki T, Ohshita T, Ueno H, Kohriyama T, Matsumoto M. Association between central systolic blood pressure, white matter lesions in cerebral mri and carotid atherosclerosis. Hypertens Res 2009; 32 869-874.

6 Debette S, Markus HS. The clinical importance of white matter hyperintensities on brain magnetic resonance imaging: Systematic review and meta-analysis. BMJ 2010; 341: c3666.

7 O'Leary DH, Polak JF, Kronmal RA, Manolio TA, Burke GL, Wolfson SK. Carotid-artery intima and media thickness as a risk factor for myocardial infarction and stroke in older adults. Cardiovascular health study collaborative research group. N Engl J Med 1999; 340: 14-22.

8 Lorenz MW, von Kegler S, Steinmetz H, Markus HS, Sitzer M. Carotid intima-media thickening indicates a higher vascular risk across a wide age range: Prospective data from the carotid atherosclerosis progression study (caps). Stroke 2006; 37: 87-92.

9 Kurata M, Okura T, Watanabe S, Higaki J. Association between carotid hemodynamics and asymptomatic white and gray matter lesions in patients with essential hypertension. Hypertens Res 2005; 28: 797-803.

10 Avolio AP, Chen SG, Wang RP, Zhang CL, Li MF, O'Rourke MF. Effects of aging on changing arterial compliance and left ventricular load in a northern chinese urban community. Circulation 1983; 68: 50-58.

11 Agabiti-Rosei E, Mancia G, O'Rourke MF, Roman MJ, Safar ME, Smulyan H, Wang JG, Wilkinson IB, Williams B, Vlachopoulos C. Central blood pressure measurements and antihypertensive therapy: a consensus document. Hypertension 2007; 50: 154-160. 